This item was submitted to Loughborough's Research Repository by the author.

Items in Figshare are protected by copyright, with all rights reserved, unless otherwise indicated.

\title{
Period multiplication and chaotic phenomena in atmospheric dielectric-barrier glow discharges
}

\section{PLEASE CITE THE PUBLISHED VERSION}

\section{PUBLISHER}

(c) American Institute of Physics

\section{VERSION}

VoR (Version of Record)

\section{LICENCE}

CC BY-NC-ND 4.0

\section{REPOSITORY RECORD}

Wang, Y.H., D.Z. Zhang, De Zhen Wang, and Michael G. Kong. 2019. "Period Multiplication and Chaotic Phenomena in Atmospheric Dielectric-barrier Glow Discharges". figshare. https://hdl.handle.net/2134/5187. 
This item was submitted to Loughborough's Institutional Repository (https://dspace.lboro.ac.uk/) by the author and is made available under the following Creative Commons Licence conditions.

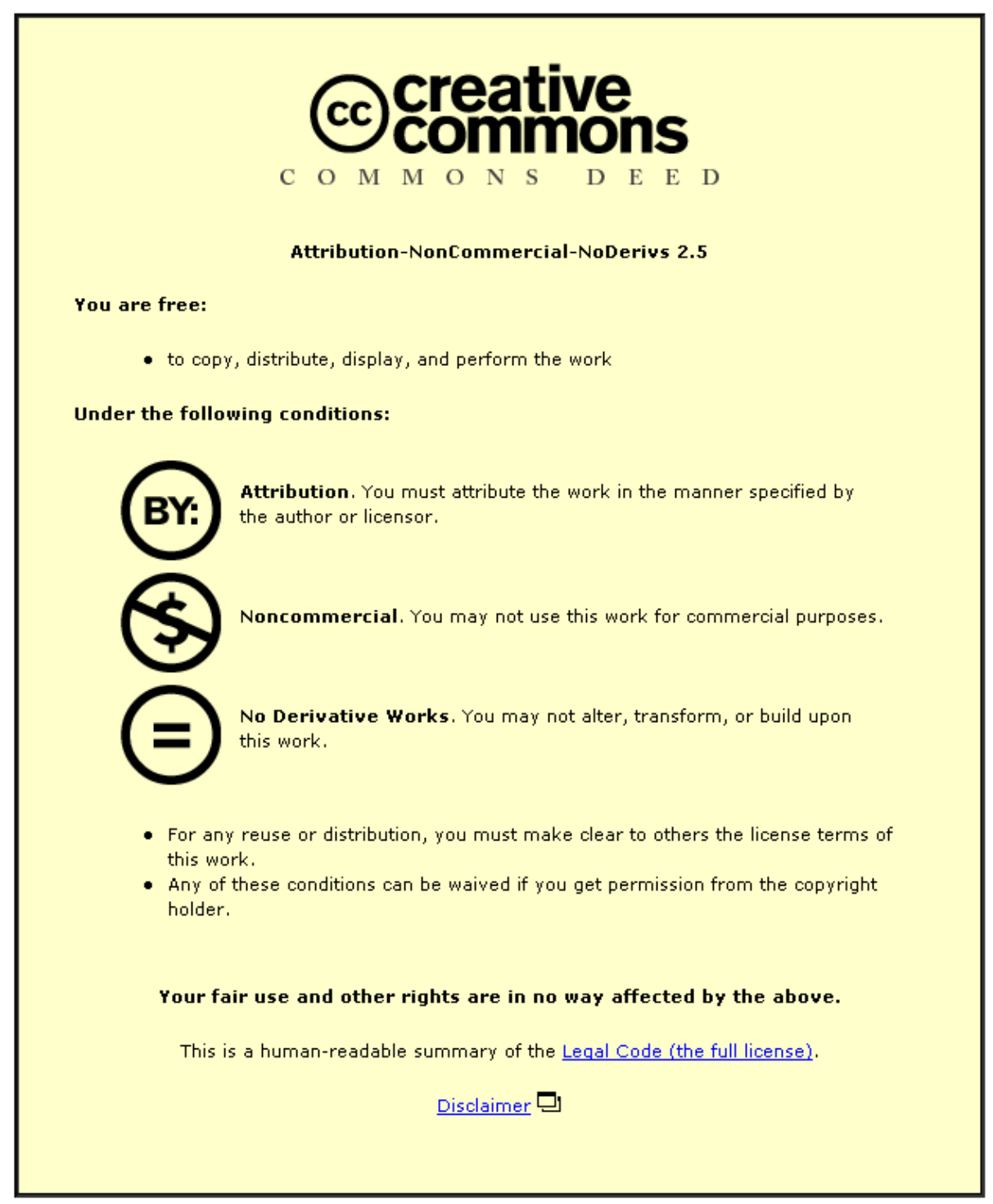

For the full text of this licence, please go to: http://creativecommons.org/licenses/by-nc-nd/2.5/ 


\title{
Period multiplication and chaotic phenomena in atmospheric dielectric-barrier glow discharges
}

\author{
Y. H. Wang, Y. T. Zhang, and D. Z. Wang ${ }^{\text {a) }}$ \\ State Key Laboratory of Materials Modification by Laser, Electron, and Ion Beams, School of Physics \\ and Optoelectronic Technology, Dalian University of Technology, Dalian 116024, China \\ M. G. Kong \\ Department of Electronic and Electrical Engineering, Loughborough University, Leics LE11 $3 T U$, \\ United Kingdom
}

(Received 7 November 2006; accepted 15 January 2007; published online 12 February 2007)

\begin{abstract}
In this letter, evidence of temporal plasma nonlinearity in which atmospheric dielectric-barrier discharges undergo period multiplication and chaos using a one-dimensional fluid model is reported. Under the conditions conducive for chaotic states, several frequency windows are identified in which period multiplication and secondary bifurcations are observed. Such time-domain nonlinearity is important for controlling instabilities in atmospheric glow discharges. (C) 2007 American Institute of Physics. [DOI: 10.1063/1.2475831]
\end{abstract}

Atmospheric pressure glow discharges (APGDs) have recently commanded much attention, largely because they offer a unique and chamberless route to many scientific disciplines. ${ }^{1-4}$ Interest in APGDs is also motivated by their scope for new low-temperature plasma physics in a highly collisional regime ${ }^{5,6}$ and by their potential as an exciting platform to study nonlinear science. ${ }^{7}$ APGDs have already been shown to possess a rich range of spatial nonlinearity, for example, self-organized pattern. ${ }^{7-9}$ In the time domain, however, a vast majority of these studies have so far been limited to APGD dynamics that follows exactly the periodic oscillation of the excitation voltage. As a spatially extended dissipated system, APGD could in principle possess complex temporal nonlinearity such as period doubling, intermittency, and chaos. ${ }^{10}$ Yet, there is at present a distinct lack of reported studies and so understanding of complex temporal behaviors and temporal nonlinearity in APGD, even though period multiplication and chaos have been observed in low-pressure discharge plasmas. ${ }^{11-14}$ A basic understanding of timedomain APGD nonlinearity is highly desirable, not only to complement the current understanding of spatial APGD nonlinearity but also to develop a basis from which to explore strategies for controlling APGD instabilities. In this letter, we report observations of complex dynamic behaviors in atmospheric dielectric-barrier discharges (DBDs) including periodic multiplication and chaos.

Our study is based on a computational approach. To focus on temporal nonlinearity without the added complexity of simultaneous spatial nonlinearity, we assume that the atmospheric DBD under study maintains uniform in the direction perpendicular to that of the externally applied voltage. The atmospheric DBD is generated by a sinusoidal excitation voltage and in a pure helium gap between two parallel-plate electrodes, each of which is covered with a thin dielectric layer. Furthermore, we assume that the gas temperature remains at $300 \mathrm{~K}$ and that plasma dynamics can be approximated by a fluid model. ${ }^{5,15,16}$ In the one-dimensional limit,

\footnotetext{
a) Author to whom correspondence should be addressed; electronic mail:
} wangdez@dlut.edu.cn densities of charged particles in the gas gap are described by the continuity equation ${ }^{16}$

$$
\frac{\partial n_{i}}{\partial t}+\frac{\partial j_{i}}{\partial x}=S_{i}
$$

where $n_{i}, j_{i}$, and $S_{i}$ represent the density, flux, and source terms for electron and ion, respectively. $j_{i}$ is deduced from the momentum equation

$$
j_{i}=\mu_{i} E n_{i}-D_{i} \frac{\partial n_{i}}{\partial x}
$$

where $E$ is the electric field. $\mu_{i}$ and $D_{i}$ are, respectively, the mobility and diffusion coefficient. Their values are taken from literature. ${ }^{17,18}$ In the source term, only the direct ionization by electron impact and electron-ion recombination in the gas gap are considered.

In our calculation, the electric field is obtained from the current conservation equation

$$
\varepsilon_{0} \varepsilon(x) \frac{\partial E(x, t)}{\partial t}+i_{c}(x, t)=i_{T}(t)
$$

where $\varepsilon_{0}$ is the vacuum permittivity. $\varepsilon(x)$ is either the relative permittivity of the dielectric layers, $\varepsilon_{B}$, or the relative permittivity of the gas gap, and so is a function of $x . i_{c}$ is the conduction current density, and $i_{T}$ is the discharge current density. The expression of $i_{T}$ is obtained by integrating Eq. (3) between the metallic electrodes. More detailed descriptions of the model can be seen in our previous article. ${ }^{16}$ The above set of equations is numerically solved in accordance with the semi-implicit Scharfetter-Gummel scheme. ${ }^{19}$

For all numerical examples presented here, the discharge gap is fixed at $1.0 \mathrm{~cm}$ and the gas pressure at 760 torr. The two dielectric layers have an identical thickness of $0.1 \mathrm{~cm}$ and an identical relative permittivity of 7.5. The secondary electron emission coefficient is set to 0.01 and the amplitude of the applied voltage to $2.8 \mathrm{kV}$. It is known that homogeneous atmospheric DBDs are characterized by one or multiple current pulses every half cycle of the applied voltage and the shape of the current pulses remains exactly the same from one cycle to another. ${ }^{7,20,21}$ This most common discharge 


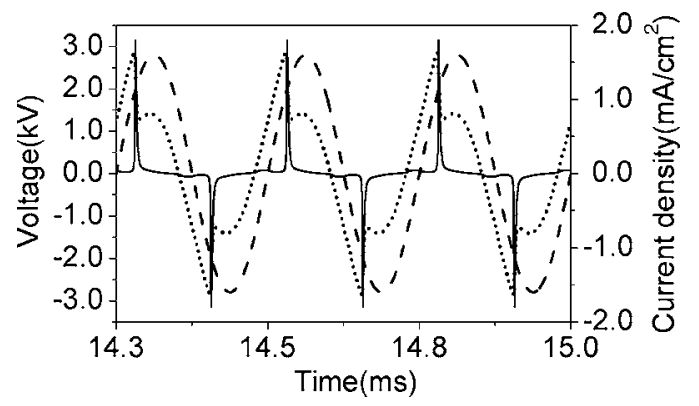

FIG. 1. Typical current density and voltage traces in an atmospheric DBD. The current density is in solid, whereas the applied voltage and the gas voltage are in dashed and dotted, respectively.

mode can be obtained over a very wide range of the excitation frequency from our numerical simulation. Figure 1 shows the temporal evolution of the discharge current in a $4.0 \mathrm{kHz}$ atmospheric DBD, together with the voltages. Figure 1 has regions where the current increases rapidly as the gas voltage reduces, implying the existence of negative differential resistance (NDR). A detailed study of NDR in atmospheric DBD is reported elsewhere. ${ }^{21}$ As reported previously, the existence of NDR indicates that the system may become chaotic under certain conditions. ${ }^{11,12}$ We explore this by increasing the driving frequency.

Figure 2 demonstrates a clear evolution from the common temporal pattern of Fig. 1, through period multiplication, and finally to chaos. As the frequency is increased to $6.55 \mathrm{kHz}$, Fig. 2(a) shows that the discharge bifurcates into a period-2 state, denoted by $2 \mathrm{P}$, in which the temporal profile of the current pulses repeats every two cycles of the applied voltage. This period doubling is clearly indicated in the two different loops in the phase space diagram in Fig. 2(a). As the excitation frequency is increased to $6.81 \mathrm{kHz}$, Fig. 2(b) shows a period- 4 state, denoted by $4 \mathrm{P}$, and its trajectory in the phase space shows four different loops. A further increase of the excitation frequency to above $6.85 \mathrm{kHz}$ results in a transition of the atmospheric DBD system into a chaotic state. At $7.10 \mathrm{kHz}$, a clear chaotic state is observed as shown in Fig. 2(c) in which the discharge behaviors are no longer periodic and the current amplitude fluctuates stochastically. It is worth mentioning that the phase plot in Fig. 2 contains

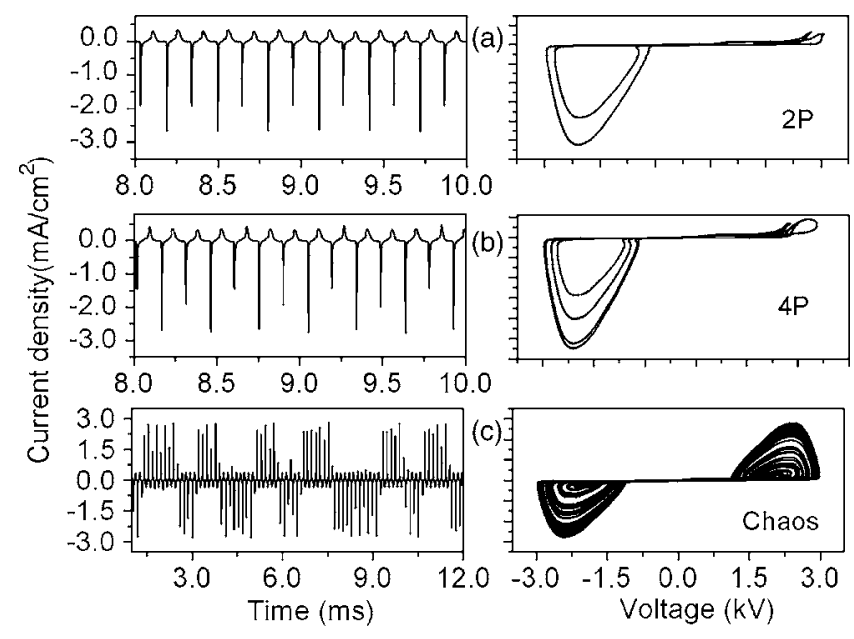

FIG. 2. Temporal evolutions of the discharge current density in (a) 2P, (b) $4 \mathrm{P}$, and (c) chaotic states together with their corresponding trajectories in the

phase space.
Downloaded 21 Aug 2009 to 158.125.80.71. Redistribution subject to AIP license or copyright; see http://apl.aip.org/apl/copyright.jsp

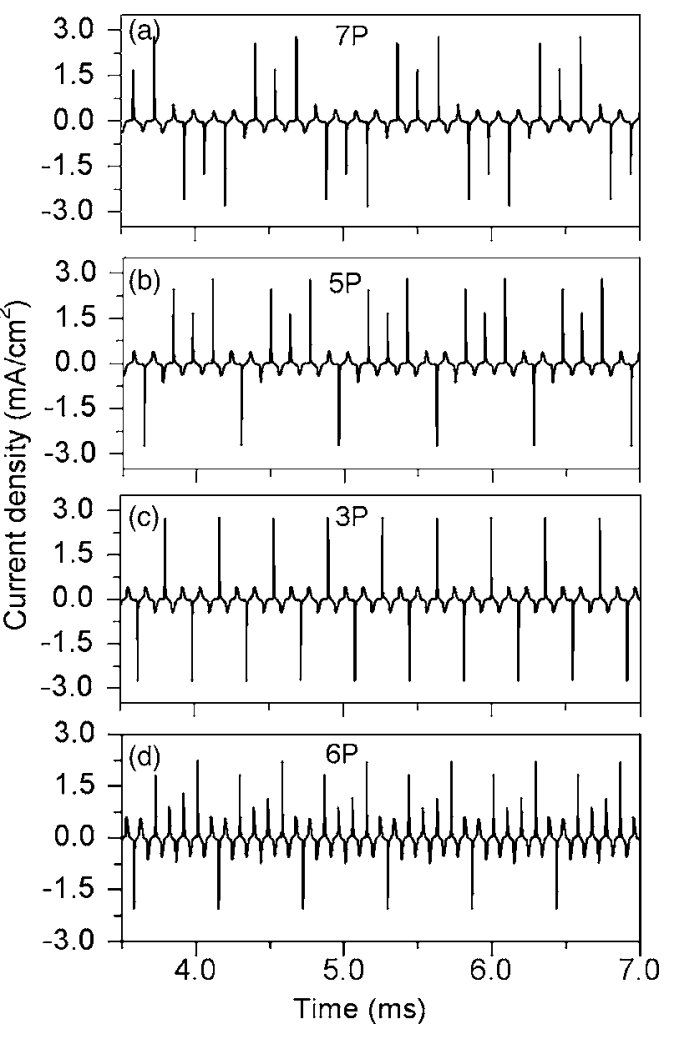

FIG. 3. Several frequency windows in a broad frequency range of prevailing chaotic DBD state.

data of 70 cycles of the applied voltage to display clearly the periodic or chaotic characters of the discharge.

After entering into the chaotic domain, we observe narrow frequency windows of period multiplication that are embedded in the broad chaos domain, as shown in Fig. 3. As the frequency is increased to $7.27 \mathrm{kHz}$, the discharge undergoes a sudden change and assumes the appearance of a period-7 state, or 7P, see Fig. 3(a). This 7P state can be maintained over a small frequency range and then is replaced by the chaotic state gradually. As the frequency is further increased, windows of 5P and 3P appear in the same way. The 5P window occupies a small frequency range centered around $7.588 \mathrm{kHz}$, whereas the $3 \mathrm{P}$ window is found to occupy a rather large frequency range. At $10.5 \mathrm{kHz}$, a period-6 state is observed as shown in Fig. 3(d). This is regarded as a secondary bifurcation from the period-3 state through period doubling. With the frequency increased above $10.5 \mathrm{kHz}$, the atmospheric DBD evolves into the chaotic state again.

Numerical data suggest that period multiplication is associated with many other changes in the characteristics of the atmospheric DBD. This can be illustrated by the period-3 case of Fig. $3(8.20 \mathrm{kHz})$ as an example. Figure 4(a) shows the temporal evolution of the discharge current density within one complete current cycle or equivalently three complete voltage cycles. There are six different current events, marked as D1, D2, .., D6. The corresponding voltages and the accumulated surface charges are plotted in Fig. 4(b). For the first discharge event of D1, the gas voltage is at its largest positive peak. Hence the gas breakdown is very strong and the resulting current pulse is very large at $2.7 \mathrm{~mA} / \mathrm{cm}^{2}$. At the peak current of D1, the spatial structure of the atmospheric DBD is shown in Fig. 5(a). This spatial structure is characteristic of a glow discharge. After the gas breakdown associated with D1 is triggered, charges accumulated on the AIP license or copyright; see http://apl.aip.org/apl/copyright.jsp 

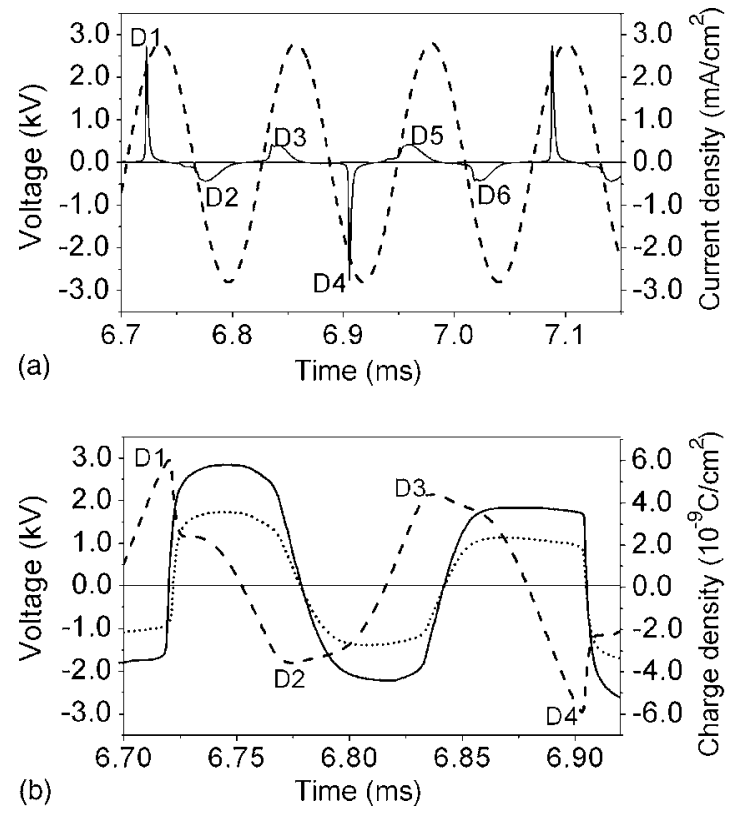

FIG. 4. (a) Temporal evolution of the current density (solid curve) in a period-3 state over three voltage cycles together with the applied voltage (dash curve), and (b) corresponding gas voltage (dash curve), memory voltage (dot curve), and surface charge density (solid curve).

dielectric layers are seen in Fig. 4(b) to maintain at a level of $6 \times 10^{-9} \mathrm{C} / \mathrm{cm}^{2}$ over the voltage-rising phase of the next half cycle of the applied voltage. In turn, the memory voltage is kept at a relatively large value of $1.75 \mathrm{kV}$, thus overly suppressing the growth in the gap voltage. As a result, the subsequent discharge event, D2, is comparatively weak. As shown in Fig. 5(b), the plasma structure of D2 deviates from the typical glow discharge. Although a sheathlike region is formed near the instantaneous cathode, this region is very broad with the zero field point extended to the middle of the gas gap. The region with quasineutrality appears to overlap with the region of zero electric field, and as such there is no positive column region. Therefore, this discharge occurs

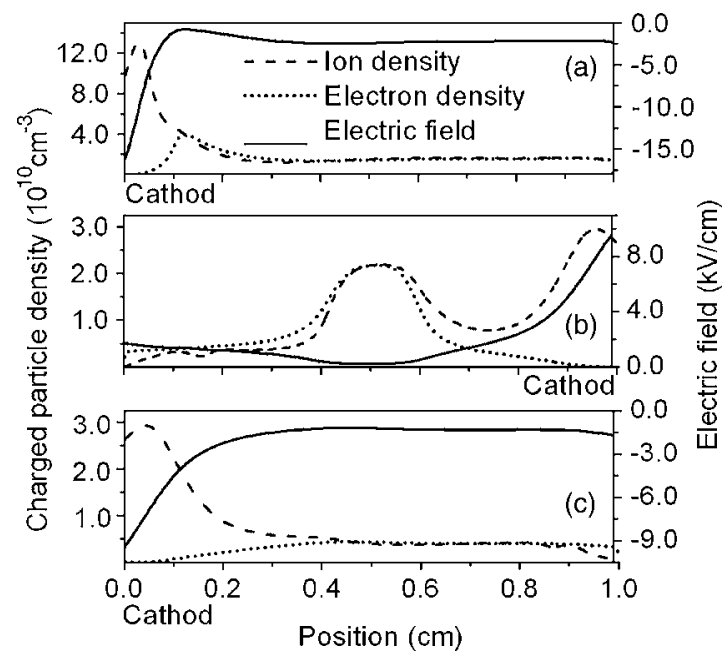

FIG. 5. Spatial structure corresponding to the discharge current pulses of D1, D2, and D3 shown in Fig. 4. when the gas voltage has not yet grown to a sufficiently large level and is, to a certain extent, a prematured discharge.

After D2, the memory voltage begins to drop and in turn this increases the gas voltage for the next gas breakdown associated with D3 to occur. Although the gas voltage is not as high as that of D1, and the discharge current density remains small, the features of glow discharge have been shown clearly, see Fig. 5(c). In the successive half cycle, the memory voltage decreases obviously and the gas voltage rises greatly. The discharge event of D4 is a fully developed one with a very similar current peak to that of D1.

It is worth mentioning that a strong discharge event of a large current is always followed by a weak and premature discharge with the same spatial structure as that of D2 whether in a period multiplication state or a chaotic state. While no data are at present available to support any concrete conclusion, we believe that one reason for premature discharges, in particular, and period multiplication is a mismatch of different time scales that are important in electron creation and loss. This mismatch could then result in a variation in the peak gas voltage from one half voltage cycle to the next and so induces different discharge current pulses in different voltage cycles. In turn, this could manifest period multiplication or even chaos in the temporal profile of the discharge currents. This should be studied in future investigations.

This work is supported by the National Natural Science Foundation of China under Grant Nos. 50537020 and 50528707 .

${ }^{1}$ D. Janasek, J. Franzke, and A. Manz, Nature (London) 442, 374 (2006). ${ }^{2}$ H. Shirai, T. Kobayashi, and Y. Hasegawa, Appl. Phys. Lett. 87, 143112 (2005).

${ }^{3}$ Y. Takao and K. Ono, Plasma Sources Sci. Technol. 15, 211 (2006).

${ }^{4}$ E. Stoffels, A. J. Flikweert, W. W. Stoffels, and G. M. W. Kroesen, Plasma Sources Sci. Technol. 11, 383 (2002).

${ }^{5}$ J. J. Shi and M. G. Kong, Phys. Rev. Lett. 96, 105009 (2006).

${ }^{6}$ J. J. Shi, X. T. Deng, R. Hall, J. D. Punnett, and M. G. Kong, J. Appl. Phys. 94, 6303 (2003).

${ }^{7}$ U. Kogelschatz, IEEE Trans. Plasma Sci. 30, 1400 (2002).

${ }^{8}$ K. H. Schoenbach, M. Moselhy, and W. Shi, Plasma Sources Sci. Technol. 13, 177 (2004).

${ }^{9}$ L. F. Dong, F. Ch. Liu, S. H. Liu, Y. F. He, and W. L. Fan, Phys. Rev. E 72, 046215 (2005).

${ }^{10}$ E. Ott, Chaos in Dynamical Systems (Cambridge University Press, Cambridge, 2002), Chap. 1.

${ }^{11}$ P. Y. Cheung, S. Donovan, and A. Y. Wong, Phys. Rev. Lett. 61, 1360 (1988).

${ }^{12}$ W. X. Ding, W. Huang, X. D. Wang, and C. X. Yu, Phys. Rev. Lett. 70, 170 (1993).

${ }^{13}$ C. Strumpel, Y. A. Astrov, and H.-G. Purwins, Phys. Rev. E 62, 4889 (2000).

${ }^{14}$ D. D. Šijaèiæ, U. Ebert, and I. Rafatov, Phys. Rev. E 70, 056220 (2004).

${ }^{15}$ M. G. Kong and X. T. Deng, IEEE Trans. Plasma Sci. 31, 7 (2003).

${ }^{16}$ Y. H. Wang and D. Z. Wang, Phys. Plasmas 12, 023503 (2005).

${ }^{17}$ R. Deloche, P. Monchicourt, M. Cheret, and F. Lambert, Phys. Rev. A 13, 1140 (1976).

${ }^{18}$ Y. P. Raizer, Gas Discharge Physics (Springer, Berlin, 1991).

${ }^{19}$ A. A. Kulikovsky, J. Comput. Phys. 119, 149 (1995).

${ }^{20}$ Yu. B. Golubovskii, V. A. Maiorov, J. Phys. D 36, 975 (2003).

${ }^{21}$ Y. T. Zhang, D. Z. Wang, and M. G. Kong, J. Appl. Phys. 100, 063304 (2006). 